\title{
Inserción al mercado del trabajo informal en jóvenes del sector turismo
}

\section{Insertion to the informal labor market in young people in the tourism sector}

\author{
Kelly Obispo-Salazar (iD) ${ }^{1}$, Lilibeth Patricia Pedraza-Álvarez (iD)2 \\ 1. Universidad del Valle. Cali, Colombia. Universidad del Magdalena. Santa Marta, Colombia. Correo: kobispo@unimagdalena.edu.co - \\ https://orcid.org/0000-0003-3618-1192 \\ 2. Universidad del Valle. Cali, Colombia. Universidad del Magdalena. Santa Marta, Colombia. Correo: Ipedrazalvarez@gmail.com - \\ https://orcid.org/0000-0002-5963-3131
}

Tipología: Artículo de investigación científica y tecnológica

Para citar este artículo: Obispo K, Pedraza A. Inserción al mercado del trabajo informal en jóvenes del sector turismo. Duazary. 2021 septiembre; $18(3$ número especial): 69-81. Doi: https://doi.org/10.21676/2389783X.4268

Recibido en abril 19 de 2021

Aceptado en julio 27 de 2021

Publicado en línea en agosto 25 de 2021

\section{Palabras clave: adulto joven; trabajo; mercado de trabajo; sector informal; empleabilidad.}

\section{RESUMEN}

El presente estudio tuvo como objetivo caracterizar la inserción al mercado de trabajo informal en jóvenes que hacen parte del sector turismo de Santa Marta, por lo cual se abordó el recurso de la empleabilidad, ya que se entiende que en la informalidad también hay estrategias, habilidades y conocimientos que inciden en el ingreso, permanencia y movilidad del trabajador. Para ello fue necesario acercarse al fenómeno desde un paradigma interpretativo, con una perspectiva cualitativa y diseño fenomenológico, el muestreo fue por caso tipo, en donde participaron nueve jóvenes entre los 19 y 27 años de edad que se dedicaban al trabajo informal tradicional menos inestable. La técnica usada fue la entrevista semiestructurada, se aplicó el análisis de contenido de Bardin y la práctica discursiva de la línea narrativa. Los resultados permiten reconocer que los jóvenes se insertan a la informalidad a través de personas cercanas, así como desarrollan habilidades sociales necesarias para ser más atractivos en este mercado evidenciándose la empleabilidad en este contexto.

\section{ABSTRACT}

Keywords: Young Adult; Work; Labor Market; Informal Sector; Employability.
The present study aimed to characterize the insertion to the informal labor market in young people who are part of the tourism sector of Santa Marta, for which the employability resource was addressed, since it is understood that in informality there are also strategies, skills and knowledge that affects the income, permanence and mobility of the worker. For this, it was necessary to approach the phenomenon from an interpretive paradigm, with a qualitative perspective and phenomenological design, the sampling was by type case, in which nine young people between 19 and 27 years of age participated who were engaged in less unstable traditional informal work. The technique used was the semi-structured interview, Bardin's content analysis and the discursive practice of the narrative line were applied. The results allow us to recognize that young people are inserted into informality through close people, as well as develop the social skills necessary to be more attractive in this market, evidencing their employability in this context. 


\section{INTRODUCCIÓN}

La juventud ha sido objeto de estudio desde diferentes campos de conocimiento ${ }^{1}$, y específicamente en la psicología se ha abordado desde una perspectiva de desarrollo, pero también en cómo son los procesos de inserción de esta población en el mercado de trabajo ${ }^{1-3}$. Por mucho tiempo, en algunos países, se catalogó como una fase de transición psicosocial hacia la adultez, que se caracterizaba por la preparación escolar y la posterior inserción al mercado de trabajo, lo cual podía estar asociado a una realidad socioeconómica y cultural destacada por la estabilidad, que fue, hasta mediados de la década de los 70 , una de las características del mercado laboral ${ }^{3}$. No obstante, la crisis del capitalismo ${ }^{4,5}$ y la reinstitucionalización del trabajo $^{6,7}$ han afectado la inserción en el mercado del trabajo de los jóvenes ${ }^{2-5}$.

Los jóvenes tienden experimentar mayor inestabilidad, desempleo y subempleo ${ }^{1,8-11}$, siendo su inserción en el mercado del trabajo complicada ${ }^{12}$, las ofertas de trabajo suelen ser informales $y$ precarias $^{13}$, de hecho, hasta antes de la pandemia generada por la Covid-19, en el mundo alrededor del 77\% de los jóvenes hacían parte del sector informal ${ }^{14}$. Particularmente en Colombia la informalidad representa una tasa de $49,2 \%{ }^{15}$, mientras que el desempleo juvenil se encuentra en un $22,5 \%{ }^{16}$, así, si se considera, como lo manifiesta $\mathrm{Neffa}^{17}$ a la informalidad como un "disfraz" del desempleo, la cifra sería de $71,7 \%$. Lo anterior estaría, entre otros motivos, conduciendo al trabajo en su modalidad informal ${ }^{18}$.

El trabajo como fundante ontológico social implica transformación ${ }^{19-25}$, y a nivel personal contribuye en la construcción de significados y la configuración de la identidad social, las características personales y profesionales ${ }^{26-29}$. El trabajar es entonces una fuente de subsistencia como de reconocimiento social, que va a incidir sobre la formación de la identidad, las relaciones en los diversos ámbitos de la vida y los significados atribuidos al trabajo ${ }^{25}$. A lo largo de los años esta actividad ha tenido cambios que han conducido a su reinstitucionalización ${ }^{7}$ y a la emergencia de nuevas modalidades ${ }^{30}$ en donde el empleo es cada vez más inestable, emergiendo formas distintas caracterizadas por la precariedad, la autogestión y la informalización ${ }^{31}$.

Producto de los cambios en el mundo del trabajo se ha hablado sobre la tesis del fin del trabajo, sin embargo, para Antunes ${ }^{32}$ no es posible referirse a un fin del trabajo, sino a la proliferación de una nueva morfología del trabajo, cada día es mayor el número de personas que trabajan desde diversas modalidades (empleo, no empleo, informal, etc.) ${ }^{33}$, entre ellas la informalidad que hace parte de la economía informal, en la que no se paga impuestos pero el trabajo realizado es legitimado socialmente ${ }^{34}$. El trabajo informal está caracterizado por la falta de acceso a la seguridad social, así como los derechos que tiene un empleado formal y a la baja o nula participación en actividades sindicales ${ }^{18}$, lo cual genera precariedad con el uso intensivo de la fuerza laboral, bajo condiciones mínimas que pueden estar relacionadas solo con la supervivencia ${ }^{35-38}$, esto, de acuerdo con el informe de los Objetivos de Desarrollo Sostenible ${ }^{39}$ 2020, tiende a tener un efecto negativo en los ingresos, el tiempo de trabajo, la seguridad y la salud ocupacional, así como en las condiciones laborales. Pese a lo anterior, hay quienes reconocen que hay ventajas en este mercado de trabajo $^{29}$, que pueden existir aspectos más allá de los asociados a la subsistencia, y que mantienen a la persona en ese trabajo, por lo que se ha generado el interés de estudiar en este mercado el constructo de la empleabilidad en el contexto del turismo en la ciudad de Santa Marta, Colombia.

La empleabilidad es un recurso que explica cómo las personas se insertan, mantienen y movilizan en el mercado del trabajo ${ }^{23,28,40,41}$. En la actualidad el mercado es variable e inestable, en cualquier modalidad de trabajo las personas pueden estar impulsadas a gestionar el desarrollo de su propio desempeño, su desenvolvimiento y el compromiso con esta actividad. Al abordar la empleabilidad se reconocen cuatro dimensiones (socioeconómica, cultural y política, institucional de la educación y de los dispositivos de formación, organizacional y de las relaciones de trabajo y la individual) que van a incluir diferentes condiciones, abordajes $y$ actores 
sociales $^{40,41}$, para este estudio se tendrá en cuenta la individual, que se centra en el análisis de los recursos, atributos y competencias de quienes hacen parte del mercado de trabajo ${ }^{41}$. En la dimensión individual se pueden identificar estrategias 0 competencias asociadas conocimientos técnicos o especializados, competencias relacionales y metacognitivas ${ }^{42}$. En la informalidad se pueden encontrar competencias que conducen a las personas a insertarse, mantenerse y movilizarse en el mercado del trabajo ${ }^{42}$, por lo anterior, algunas personas pueden ser más atractivas que otras en cuanto al contexto de trabajo ${ }^{29}$, que, para el caso de este estudio, son los jóvenes en el sector turismo de la ciudad de Santa Marta.

Hasta el año 2019 el turismo se destacaba por su rápido crecimiento ${ }^{43}$, siendo uno de los sectores con mayor generación de empleos directos e indirectos, sobre todo en la población de mujeres y jóvenes ${ }^{14}$. El turismo se caracteriza por ser uno de los sectores que presenta mayores tasas de informalidad $69,67 \%$, teniendo un alto impacto en los alimentos y bebidas, a pesar de ello, es un sector que ha representado el $7,78 \%$ del empleo en el país ${ }^{44}$. Particularmente en Santa Marta, el turismo se encuentra el segundo puesto de mayor informalidad $^{45}$, en donde se pueden encontrar actividades relacionadas con la tipología propuesta con Antunes ${ }^{31}$ y Alves y Tavares ${ }^{46}$, a los trabajadores informales menos inestables, entre ellos quienes se dedican a ventas informales como promotores, informadores y guías turísticos. Por lo anterior se propone caracterizar la inserción al mercado de trabajo informal en jóvenes que realizan esta actividad en el sector turismo de Santa Marta desde la empleabilidad.

\section{MATERIALES Y MÉTODOS}

\section{Diseño}

Este estudio se abordó desde una perspectiva cualitativa, ya que se buscó especificad y no replicabilidad $^{47}$, el análisis de las versiones del mundo ${ }^{48}$, a partir de una aproximación naturalista e interpretativa ${ }^{49}$, enmarcado en un paradigma interpretativo ${ }^{50}$. El diseño es el fenomenológico, lo que indica que se describió el fenómeno en un sentido amplio, tal y como aparecía en la conciencia de quien lo experimentaba ${ }^{51}$, en este caso el proceso de inserción en el mercado de trabajo de los jóvenes.

\section{Participantes}

Los jóvenes tenían entre 19 y 27 años de edad, para lo cual se tuvo en cuenta la Ley Estatutaria 1662 de 2013. El tipo de muestreo aplicado fue de caso tipo, dado que se dio prelación a la riqueza, profundidad, vista de forma multidimensional, y a la calidad de la información ${ }^{52}$ que aportaban los jóvenes en un marco geográfico y el sector de trabajo específico, en este sentido, el total de participantes fue de nueve, ocho hombres y una mujer. Además de la edad, se tuvo en cuenta como criterio de inclusión el tipo de actividad informal desde la propuesta de Antunes ${ }^{31}$, Alves y Tavares ${ }^{46}$ sobre los trabajadores informales, siendo trabajadores informales tradicionales, menos inestables, que pertenecen al sector de productos o servicios, para este caso a la venta y guíanza de planes turísticos.

\section{Técnicas de recolección de información}

Se hizo uso de la entrevista con el propósito acercarse a la realidad de los participantes, buscando profundizar en tiempo real sobre tópicos de interés y de relevancia para la comprensión del fenómeno estudiado ${ }^{53}$. Particularmente se aplicó la entrevista semiestructurada en la que el investigador define previamente los tópicos a tratar con el entrevistado, además, se pueden formular preguntas que no estaban previstas ${ }^{54}$. En total se hicieron nueve entrevistas, seis de la cuales se realizaron por medio de la plataforma ZOOM 5.4 .9 y tres presencialmente. En tres ocasiones se hizo una segunda entrevista virtual para aclarar aspectos de la primera y confirmar otros relacionados con el análisis inicial.

\section{Análisis de información}

Se aplicó el análisis de contenido desde la perspectiva de $\operatorname{Bardin}^{55}$, que hace referencia a la aplicación de un conjunto de técnicas de análisis de comunicaciones para obtener indicadores mediante procedimientos sistemáticos y objetivos que 
permiten la descripción del contenido de las narraciones, así como la inferencia de conocimientos asociados al fenómeno de estudio. Para conocer "lo que se cuenta" se tomaron como unidades de registro el tema, el personaje y el acontecimiento. Las reglas de enumeración "la manera de contar" aplicadas fueron la frecuencia, para evidenciar la importancia de aparición de las unidades, y la coocurrencia, para comprender las implicaciones que hay entre los datos. Igualmente, se hizo uso de las prácticas discursivas ${ }^{56}$, específicamente del árbol de asociación de ideas, reconociendo en las narraciones de los participantes argumentos asociados entre sí para analizar la forma en que se visibiliza el problema de estudio. El análisis de la información se hizo a través del Software para datos cualitativos Atlas.ti 9.1.0.

\section{Medidas de validez y fiabilidad}

Se usó el método de comprobación de coherencia con el objetivo de garantizar la validez y fiabilidad de los resultados, por lo que se solicitó a otro codificador la revisión y análisis de las categorías creadas $^{57}$.

\section{Declaración sobre aspectos éticos}

Antes de iniciar cada entrevista se presentó el consentimiento informado en el que los participantes autorizaban ser grabados para recobrar la información posteriormente, así como se garantizaba la confiabilidad de los datos personales. De acuerdo a la Resolución 8430 de 1993 del Ministerio de Salud de Colombia el estudio no representa ningún riesgo para los participantes, siendo una investigación sin riesgo.

\section{RESULTADOS}

Con el propósito de caracterizar los aspectos sociodemográficos asociados a la inserción al mercado de trabajo informal de los jóvenes participantes, se pudo reconocer que cuatro de los nueve jóvenes del estudio ingresaron a dicho mercado antes de los 18 años de edad. El rango de edad de los jóvenes fue de 19 a 27 años, y, solo una de las participantes pertenece al sexo femenino, el resto pertenece al masculino. En cuanto al nivel de formación cinco de los jóvenes son bachilleres, tres tecnólogos y uno no termino el bachillerato; tres participantes viven en unión libre y los demás son solteros.

Desde el recurso de la línea narrativa se presentan los argumentos organizados por parte de los participantes que permiten reconocer el proceso de inserción en el mercado de trabajo informal, sus características y factores representativos destacados por los participantes (Figura 1).

El análisis de contenido se usó para explicar los factores asociados al proceso de inserción en el mercado laboral, por lo cual se utilizó la regla de enumeración de coocurrencia, así como las unidades de registro de tema y personaje, esta regla permite reconocer las implicaciones que tienen las unidades de registro entre sí. Se encontró que uno de los factores para el proceso de consecución e ingreso al trabajo informal es la recomendación que hacen los amigos y familiares, sobre lo cual expresaban "...cuando termino el bachiller que me graduó, es donde Andrés dice $<<n o$ que estás haciendo $>>,<<n o$ acabe este año ya será otro sexto>>, él me dice <<nombre, vámonos para el Rodadero, te voy a enseñar como animal las chivas para que tu veas más que se pasa bacano generas ingresos > ..." (P4), "Una amiga, una amiga me lo recomendó (...) ella me recomendó acá, vio que mi potencial y hasta el sol de hoy estoy aquí..." (P7).

Otros personajes importantes en el proceso de inserción son los tíos "Entonces, yo cuando venía de vacaciones de Medellín, ellos me decían <<bueno mira, tú no estás en vacaciones, aprovechas que estas aquí y te llevas tus cositas para Medellín>> y yo <<bueno dale, tío dale >>. Yo me iba con ellos, ya ellos me decían <<muy pendiente con el turista... al turista primero prestarle mucha atención y tenerle mucha paciencia >>..." (P3), las mamás "...entonces desde ahí mi mamá empezó como que por qué no te llevas a María y le enseñas..." (P1). Se destaca el factor económico para la inserción "yo venía a vacaciones, y mi tío, no ósea, yo no venía a descansar, yo venía a trabajar, para ganarme mis cosas y todo..." (P3) y la salud "...pues tuve una lesión en la rodilla y duré seis meses sin entrenar y 
cuando me recuperé no estuve el $100 \%$ y pues me

retiré y empecé con el turismo" (P6).

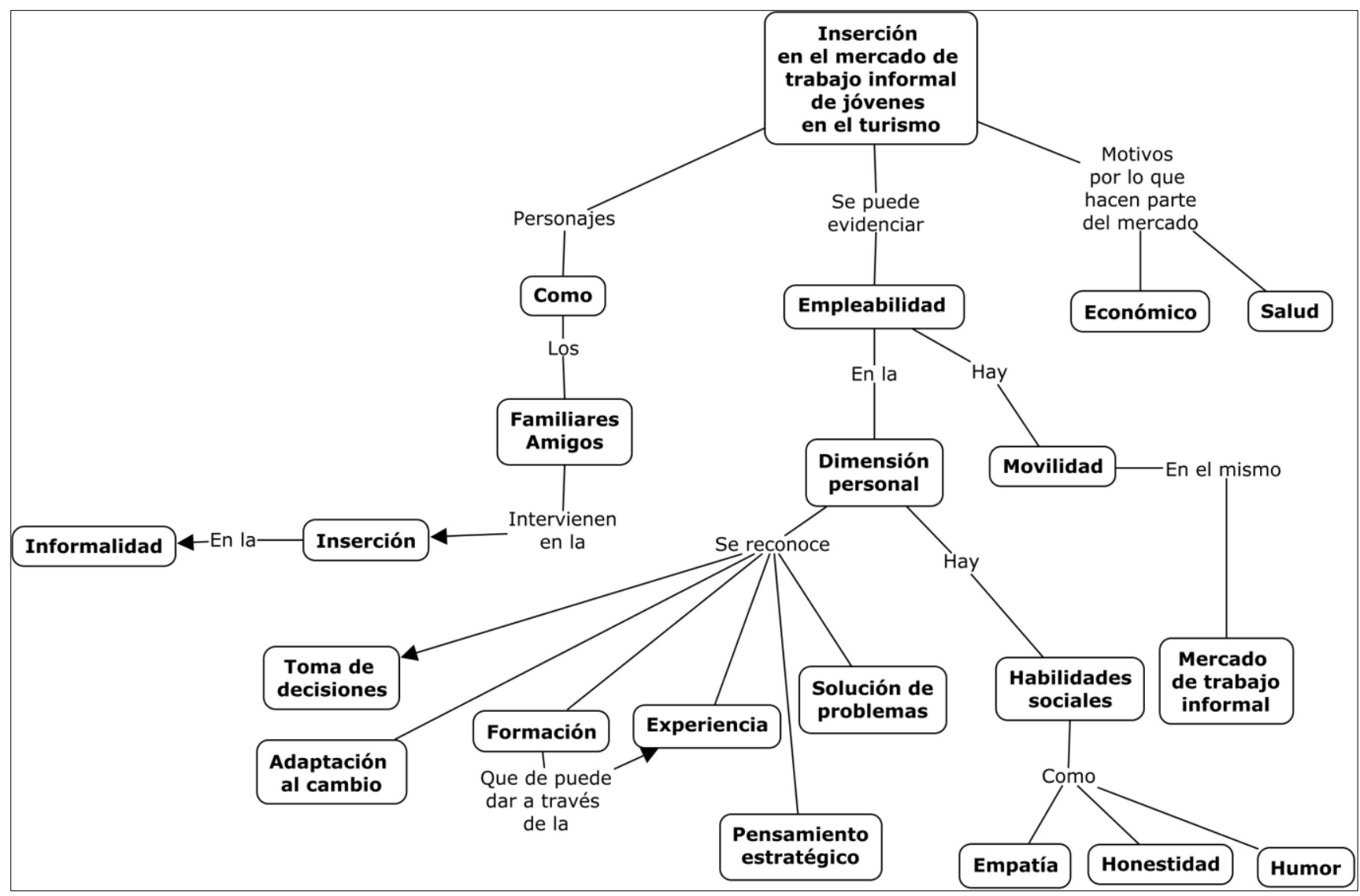

Figura 1. Árbol de asociación de ideas sobre la inserción en el mercado de trabajo informal de jóvenes en el sector turismo.

La inserción al mercado del trabajo informal también se explicó desde el recurso de la empleabilidad, siendo esta la categoría de estudio con la subcategoría de recursos personales. Entre los indicadores con mayor cantidad de apariciones estaban Formación (71), Habilidades sociales (59), Experiencia (33), Pensamiento estratégico (31), Movilidad en el mercado de trabajo (23), Solución de problemas (17), Adaptación al cambio (11) y Toma de decisiones (6).

En cuanto al indicador de formación los participantes señalaban que es necesario tener conocimientos sobre la historia de la ciudad, saber sobre el lugar a donde llevan a los turistas, "Lo único es que desde el primer momento debes tener bastante conocimiento de la ciudad, y sobre el lugar al que estas yendo..." (P1), "Si la formación es saberte toda la historia de los sitios turísticos, saberte la historia, de donde viene, de qué proviene..." (P2), además, explicaban que era necesario para este tipo de trabajo "... una formación que tu sepas que es lo que tienes que hacer, no solamente lo que aprendas, sino que tu vayas formándote poco a poco y vayas aprendiendo como tratar al cliente (...) que hagas un curso..." $(P 3)$, sin embargo, era una condición que ellos previamente como informales no tenían, lo recomendaban para otros, pero su conocimientos y saberes eran producto de la experiencia en el campo.

Entre las habilidades sociales que destacan los jóvenes para el desarrollo de la actividad están la empatía, la honestidad, así lo expresaban "tener buena comunicación, empatía, ser también muy empático; honesto..." (P1) además de ser "Abierto, ser empático, o sea tener la posibilidad de ponerse, pues, en el papel del otro... <<esta persona si yo la trato, como dices tú, si yo la trato de determinada 
manera, se podría sentir de determinada manera entonces $>>$ " (P2), también se requiere de buen humor "...súper sociable y relacionarse con el turismo, porque si la persona si le cae mal, o sea sino tienen por ejemplo, sentido del humor, no tiene una capacidad buena de venta, no, no sirve para este trabajo" (P8), de ser propositivo "Me van a decir $<<(.$.$) , necesito una cerveza o algo >>,<<j e f e$, ya se la busco >..." (P3) , carismático "...aparte del carisma, o sea, como el saber... el saber, por decirlo, leer la clase de turista, porque uno tiene que saber a quién le entra con carisma, a quien le entra con personalidad, a quien le entra con humor, porque uno nota..." (P4) "...ser carismáticos es importante y tener un buen léxico" (P7).

El trabajador se caracteriza por generar confianza "Pues directamente acá lo primordial para ser un buen vendedor es no tener (...) miedo como tal a la hora de ofrecer los paquetes, ni a la hora de acercársele al turista directamente siempre hablar tranquilo..." (P9), así como hacer parte de ese tipo de trabajo contribuye en la creación de relaciones sociales "también por cómo te dije crear nuevas relaciones" (P1).

Para los participantes la experiencia se adquiere al momento de ingresar al mercado "hasta que la compañera me dijo <<no, te toca y empieza que yo te ayudo >> y yo le decía <<¿Cómo? pero si yo todavía no sé>><<empieza que yo te sigo y te ayudo>> y así fue como me le medí, y ya después de ahí..." (P2) algunas veces la experiencia se adquiere en compañía de otros trabajadores en el campo "...luego, prácticamente como a los tres, cuatro meses yo me iba con un guía profesional, o sea yo me iba como si fuera un turista pero ahí iba 6-7 personas podrían ser más o menos, si me entiende, podrían ser 6- 7 personas que podía llevar yo ahí y yo me iba para ir aprendiendo, o sea cómo se da una guianza, qué era lo que había que brindarle al turista..." (P4) no hay un proceso de formación previo porque "...Como decirlo uno, todo to he aprendido en la calle" (P2).

Los jóvenes tienden a organizar sus actividades, en ellas piensan estratégicamente, solucionan problemas y se adaptan al cambio "...no, yo siempre iba era a vender era los planes, (...) uno iba caminando (...) uno iba por la calle, veía al turista. Ya eso era como... una casualidad... ya eso era como una casualidad, eso era como uno ver al turista por oportunidad, y uno la aprovechaba..." (P4) en cuanto a solucionar problemas en las que no se dominaba el idioma inglés, los jóvenes manifestaban "pues directamente cuando yo no sé inglés, ya uno usa cualquier otra herramienta de repente ehhhh de repente con el celular uno con traductor u otro compañero..." (P9).

También deben tomar decisiones para buscar mejores opciones de servicios "Ok, bueno... listo, eh, empecé fue como Decameron. En Decameron uno trabaja en varios sectores, ya sea el Rodadero, Taganga, Playa... Blanca y Bahía Concha no lo habían habilitado, porque para habilitarlo tienen que darle como unos viáticos a uno. Total, que como no lo habían habilitado, yo por cuenta mía decidí abrir esa locación, empezar a ir sin nada, sin viáticos (...) o sea mirar yo como almorzaba, como resolvía yo mi..." (P2).

Por último, se observa que los participantes tienen la posibilidad de cambiar la oferta de servicios en el mismo mercado de trabajo, de realizar otras actividades en el sector turismo, sin dejar de ser informales "incluso tu ves a una persona que se baja de un bus, un turista, o de un taxi, con maletas y todo, tu te le acercas: <<no... te tengo el hotel, uno

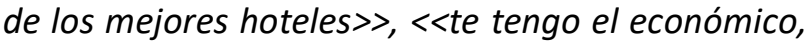
te tengo a tu gusto, te tengo a tu alcance, te tengo lo que tu quieras" entonces el turista te dice $<<s i$ claro, muéstrame que hotel me puedes recomendar >> ..." (P4).

\section{DISCUSIÓN}

El objetivo de este artículo fue caracterizar la inserción al mercado de trabajo informal en jóvenes que realizan esta actividad en el sector turismo de Santa Marta, desde el recurso de la empleabilidad. Para esto fue necesario reconocer factores de carácter sociodemográfico como las edades y el nivel de formación de los participantes, ya que los jóvenes entre 15 y 19 años de edad tienden a ingresar al mercado de trabajo informal, entre otros motivos por los bajos niveles de educación que se correlacionan negativamente con esta modalidad de 
trabajo ${ }^{9}$, y es que precisamente la condición de ser joven en América Latina, de acuerdo a Gallardo ${ }^{1}$ esta asociada a situaciones y problemas como la falta de empleo, la precariedad laboral, la falta de oportunidades educativas, la pobreza, etc., hechos que afectan a la sociedad en general, pero que hacen más vulnerable a esta población. En esta línea en el informe de los Objetivos de Desarrollo Sostenible del año $2019^{58}$ se reconoce que una quinta parte de los jóvenes no cursan estudios, ni trabajan o reciben formación.

Los participantes de este estudio ingresaron al mercado de trabajo informal a través de personas cercanas, amigos y familiares, eran estos personajes quienes los motivaban a realizar actividades dentro del sector turístico y bajo la modalidad informal. Es probable que aquellos que trabajan en la informalidad se inserten por la influencia de personas de su alrededor ${ }^{59,60}$. De acuerdo con Busso $^{61}$ las personas pueden ejercer este tipo de actividades por herencia, en varias generaciones. La inserción y el mantenimiento en la informalidad esta mediada por experiencias cercanas en dicho mercado lo que influye sobre la toma de decisión de hacer parte de él.

Parte de la reflexión de este estudio, es que, si bien en la mayoría de los casos la inserción al mercado informal puede ser precaria y marcada por bajos niveles de formación, las personas, y particularmente los jóvenes participantes, tienden a permanecer en él, ya que encuentran ventajas a nivel económico, de estatus, flexibilidad $y$ autonomía, específicamente se valora la actividad realizada considerando las ganancias monetarias que provee ${ }^{18}$. En este sentido, se reconoce el aspecto extrínseco o instrumental del trabajo, en cuanto es un instrumento de remuneración económica ${ }^{62}$. Los jóvenes se insertan por motivos económicos, pero también logran mantenerse e inclusive movilizarse en ese mismo mercado dado los beneficios económicos que genera este tipo de actividad.

Para el desarrollo del trabajo informal de guía, promotor o informador, los jóvenes entrevistados no tenían una formación profesional o especializada previa para ejercerlo, no obstante, por las condiciones de este tipo de trabajo, es posible encontrar una cualificación específica sobre historia, cultura y atención al cliente $^{63}$, saberes que se aprenden en el día a día ${ }^{64}$, es decir, en este contexto las personas tienen conocimientos y saberes necesarios para el desarrollo de su actividad ${ }^{61}$, pero son adquiridos en su quehacer diario. De acuerdo a lo encontrado, el no estar cualificado, en términos de formalización académica, no es un impedimento para insertarse y mantenerse en este trabajo. La experiencia es un factor que favorece los conocimientos y saberes ${ }^{63}$.

En este tipo de actividad el trabajador aprende habilidades sociales para el desarrollo de su trabajo, se reconoce que ser empatíco es muy importante, así como tener buen humor y leer el contexto, para identificar cuál es la mejor manera de acercarse a ellos logrando compra exitosa del servicio que ofrecen. Este es uno de los aspectos que se reconoce como ventaja de la informalidad, pues el trabajador desarrolla habilidades que van a facilitar la comunicación con los turistas ${ }^{63,64}$. Igualmente, el generar confianza a partir de esas habilidades sociales, conocimientos y experiencia en el mercado, es uno de los aspectos que hace que las ventas de toures y servicios turísticos sean más exitosas.

Por lo general, al referirse a la informalidad se hace como antagónico de formalidad ${ }^{36}$, se asumen como antónimos, entre lo regulado y lo no regulado ${ }^{65}$, en ese sentido, el trabajo informal se asocia, entre otros, a carecer de forma u organización, no obstante, los resultados de este estudio, así como los hechos por Andrade, et $\mathrm{al}^{60}$, Xavier, et $a l^{63} \mathrm{y}$ Sato $^{64}$, permiten reconocer que los trabajadores bajo esta modalidad implementan estrategias para organizar su trabajo, lo que les ayuda a solucionar problemas, tomar decisiones y adaptarse a los cambios, esto los hacía mucho más atractivos para el mercado de trabajo, ya que podían aumentar el número de clientes, de ventas de servicios.

El turismo se caracteriza por altas tasas de informalidad, y por la heterogeneidad de trabajos que bajo esa modalidad se realizan, por eso se pueden encontrar personas que se movilicen, esto producto de los intereses a nivel económico y psicosociales que representa el trabajo para las personas. El trabajo representa un factor de 
socialización ${ }^{66}$, de construcción de identidades ${ }^{7}$, de estatus, de autonomía ${ }^{21,62}$, de reconociento y transformación. En la actualidad, por los cambios que se han generado en el mundo del trabajo, recursos como la empleabilidad se hacen visibles para abordar los procesos de ingresos al mercado de trabajo, así como su permanencia y movilidad, y no solo dentro de contextos formales, sino informales que hoy son más recurrentes en países de América Latina.

En este artículo fue posible caracterizar la inserción al mercado informal por parte de jóvenes en el sector turismo de Santa Marta, ello explicado desde la empleabilidad, como recurso aplicado a la informalidad, en donde se pueden desarrollar estrategias y habilidades sociales, aplicar conocimientos sobre temas particulares, que hacen al joven trabajador más atractivo para el mercado de trabajo. Por tanto, se concluye que puede existir empleabilidad en el contexto informal, y en ello incide, entre otros factores, la cercanía de personas que también trabajan en él, por lo que se propone que, más allá de cuantificar el fenómeno de la informalidad, propio de entes como la OIT y el DANE, para Colombia, será preciso abordar la complejidad de dicho fenómeno desde la narrativa de quienes hacen parte de él, quizá así serán las políticas y los objetivos de desarrollo sostenible más efectivos y coherentes con la realidad del trabajador informal. Se recomienda realizar investigaciones desde una perspectiva cuantitativa para ampliar la caracterización hecha en este estudio, y así contribuir desde el campo de la psicología de las organizaciones y del trabajo a la heterogeneidad del mercado laboral actual.

\section{AGRADECIMIENTOS}

Agradecimiento al Ministerio de Ciencia, Tecnología e Innovación por el apoyo a través de las Beca de Excelencia Doctoral del Bicentenario corte I. Asimismo, a los participantes por contribuir a la realización del proyecto.

\section{DECLARACIÓN SOBRE CONFLICTOS DE INTERESES}

Los autores declaran que no existe conflicto de intereses en la realización de la presente investigación.

\section{CONTRIBUCIÓN DE LOS AUTORES}

Primer autor: trabajo de campo, diseño metodológico, análisis de la información y redacción;

Segundo autor: análisis de la información, redacción.

\section{REFERENCIAS BIBLIOGRÁFICAS}

1. Gallardo J. Youth, work, unemployment and identity: An social psychological approach. Athenea Digital. $2011 \quad$ Nov;11(3):165-182. Doi: https://doi.org/10.5565/rev/athenead/v11n3.898

2. Coelho R, Álvaro J, Garrido A. Juventud alargada y trabajo: desafíos del mundo laboral en las experiencias de jóvenes brasileños y españoles. 2014 Oct;14(4):417-427. Disponible en: http://pepsic.bvsalud.org/pdf/rpot/v14n4/v14n4a0 8.pdf

3. Paulino D, Bendassolli P. Significado del trabajo y de la búsqueda de empleo para jóvenes ninis. Avances en Psicología Latinoamericana. 2018;36(2):373-388. Doi: http://dx.doi.org/10.12804/revistas.urosario.edu.c o/apl/a.5112

4. Harvey D. Condição pós-moderna: Uma pesquisa sobre as origens da mudança cultural. São Paulo: Editorial Loyola; 2009.

5. Blanch J. La juventud nini, un agujero negro psicosocial. Revista Psicología: Organizações e Trabalho. 2014 Oct;14(4):355-366. Disponible en: http://pepsic.bvsalud.org/pdf/rpot/v14n4/v14n4a0 3.pdf

6. Rentería E. Desarrollo de la empleabilidad como estrategia para las organizaciones: limitaciones, posibilidades e implicaciones para las personas. Revista Aristeo. 2012;2:69-90. Disponible en: https://www.researchgate.net/publication/337800 935_Desarrollo_de_la_empleabilidad_como_estrat 
egia_para_las_organizaciones_limitaciones_posibili dades_e_implicaciones_para_las_personas

7. Rentería E. Psicología (s) Organizacional (es) y del (de los) Trabajo (s). Coexistencia de realidades e implicaciones disciplinares y para las personas. Un re-introducción. Cali: Programa Editorial de la Universidad del Valle; 2019. Disponible en: https://webcache.googleusercontent.com/search? $\mathrm{q}=$ cache:UBEJ5IvBWfOJ:https://libros.univalle.edu.c o/index.php/programaeditorial/catalog/book/458+ $\& c d=2 \& h l=e s \& c t=c \mid n k \& g l=c o \& c l i e n t=s a f a r i$

8. Organización internacional del trabajo. Panorama 2012 Laboral América Latina y el Caribe. [internet]. [Consultado 2021 Abri 20]. Disponible en: https://www.ilo.org/wcmsp5/groups/public/--americas/---ro-

lima/documents/publication/wcms_195884.pdf

9. Organización internacional del trabajo. Trabajar para un futuro más prometedor. 2019. Disponible en: https://www.ilo.org/wcmsp5/groups/public/--dgreports/---

cabinet/documents/publication/wcms_662442.pdf

10. Jiménez D. La informalidad laboral en América Latina: ¿explicación estructuralista o institucionalista? Cuadernos de Economía. 2012 Jul;31(58):113-143. Disponible en: https://www.redalyc.org/pdf/2821/282125048006. pdf

11. Torres-López $T$, Acosta-Fernández $M$, AguileraVelasco M. Preparación para el trabajo: Representaciones sociales del empleo y desempleo de jóvenes mexicanos sin trayectoria laboral. Revista Educación y Desarrollo Social. 2017; 11(1):85-101.

http://dx.doi.org/10.18359/reds.1867

12. Finkel L. La organización social del trabajo. Madrid: Pirámide; 1994.

13. Coelho R. El Alargamiento de la Juventud: un análisis psicosocial de las trayectorias de jóvenes en Brasil e España. Madrid: Universidad Complutense de Madrid; 2013.Disponible en: https://eprints.ucm.es/id/eprint/22273/1/T34558. pdf
14. Organización Internacional del Trabajo. The impact of COVID-19 on the tourism sector. [internet]. [Consultado $2021 \mathrm{Abr}$ 21]. Disponible en: https://www.ilo.org/wcmsp5/groups/public/--ed_dialogue/---

sector/documents/briefingnote/wcms_741468.pdf

15. Departamento Administrativo Nacional de Estadística Colombia Medición de empleo informal y seguridad social - Trimestre noviembre 2020 enero 2021. [internet]. [Consultado $2021 \mathrm{Abr} 9$ ]. Disponible en: https://www.dane.gov.co/files/investigaciones/bol etines/ech/ech_informalidad/bol_geih_informalida d_nov20_ene21.pdf

16. Departamento Administrativo Nacional de Estadística Colombia. Mercado laboral de la juventud trimestre noviembre 2020 - enero 2021. [internet]. [Consultado $2021 \mathrm{Abr}$ 9]. Disponible en: https://www.dane.gov.co/files/investigaciones/bol etines/ech/juventud/Boletin_GEIH_juventud_nov2 0_ene21.pdf

17. Neffa J. Sector informal, precariedad, trabajo no registrado. Congreso nacional de estudios del trabajo. [internet]. [Consultado $2021 \mathrm{Abr}$ 9]. Disponible en: https://www.aset.org.ar/congresos/9/Ponencias/p 8_Neffa.pdf

18. Coelho-Lima F, Bendassolli P. Trabalhadores e Trabalhadoras na Informalidade: Intervenções Possíveis. En: Melissa Machado. O trabalho e as medidas de contenção da COVID-19. Contribuições da Psicologia Organizacional e do Trabalho no contexto da pandemia. Sao Paulo; Artmed; 2020. p. 35-44.

19. Marx K. Capital: Libro I. México D. F.: Siglo veintiuno; 1979.

20. Peiró J, Prieto. (Eds.). Tratado de psicología del trabajo. Volumen I: actividad laboral en su contexto. Síntesis; 1996.

21. Blanch J. Psicología Social del Trabajo. En: José Luis Álvaro, Alicia Garrido, José Torregrosa (coords.), 
Psicología Social Aplicada. Madrid: McGraw-Hill; 1996. p. 85-120.

22. Antunes R. La centralidad del trabajo hoy. Papeles de población. 2000; 6(25):83-96. Disponible en:

https://www.redalyc.org/pdf/112/11202505.pdf

23. Rentería E. Empleabilidad: una lectura psicosocial. Revista de Estudios del Trabajo en Colombia. 2005;1(1):1-14. Disponible en: https://www.researchgate.net/profile/Erico_Rente ria2/publication/264869527_EMPLEABILIDAD_UNA _LECTURA_PSICOSOCIAL/links/564291e508aeacfd8 9384080/EMPLEABILIDAD-UNA-LECTURAPSICOSOCIAL.pdf

24. Tolfo S, Coutinho M, Almeida A, Baasch D, Cugnier J. Revisitando abordagens sobre sentidos e significados do trabalho. Anais do Fórum Criteos. $2005 . \quad$ Disponible en: https://www.researchgate.net/publication/215548 982_Revisitando_abordagens_sobre_sentidos_e_si gnificados_do_trabalho

25. Dutra L, Koller S. O significado do trabalho na visão de jovens brasileiros: uma análise de palavras análogas e opostas ao termo" trabalho". Revista Psicologia Organizações e Trabalho. 2014 Dic;14(4):67-380. Disponible en: http://pepsic.bvsalud.org/scielo.php?script=sci_art text\&pid=S1984-66572014000400004

26. Blanch J. Del viejo al nuevo paro. Un análisis psicológico y social. Biblioteca Universitaria de Ciencias Sociales. Barcelona: Promociones y publicaciones universitarias S.A; 1990. Disponible en:

https://books.google.com.co/books?id=jDtsAAAAC AAJ\&dq=Del+viejo+al+nuevo+paro.+Un+análisis+ps icológico+y+social.+Biblioteca+Universitaria+de+Cie ncias+Sociales\&hl=es\&sa=X\&redir_esc $=y$

27. Méda D. ¿Qué sabemos sobre el trabajo? Revista de trabajo. 2007; 3(4):17-32. Disponible en: http://www.trabajo.gob.ar/downloads/estadisticas /2007n04_revistaDeTrabajo.pdf
28. Rentería E. Nuevas realidades Organizacionales y del Mundo del Trabajo: Implicaciones para la Construcción de la Identidad o del Sujeto. Informes Psicológicos. 2008 Ene; (10): 65-80. Disponible en: https://revistas.upb.edu.co/index.php/informespsi cologicos/article/view/931/755

29. Andrade V. Identidad profesional y el mundo del trabajo contemporáneo. Reflexiones desde un resumen de caso. Athenea Digital. Revista de pensamiento e investigación social. 2014 Jul;14(2):117-145. Disponible en: https://atheneadigital.net/article/view/v14-n2andrade/1143-pdf-es

30. Antunes R. El caracol y su concha: ensayo sobre la nueva morfología del trabajo. Revista Sociología del Trabajo. 2007;59:131-142. Disponible en: https://www.nodo50.org/cubasigloXXI/congreso06 /conf3_antunes.pdf

31. Antunes R. O privilégio da servidão: o novo proletariado de serviço na era digital. São Paulo:Boitempo Editorial; 2018. Disponible en: https://books.google.es/books?hl=es\&lr=\&id=gKJfD wAAQBAJ\&oi=fnd\&pg=PT\&\&dq=0+privilégio + da + se rvidão:+o+novo+proletariado+de+serviço+na+era+ digital\&ots=75atyXM5FZ\&sig=3bRCfnNJ-

_N10zifBvlqck_E7e4\#v=onepage \&q=0\%20privilégio \%20da\%20servidão\%3A\%20o\%20novo\%20proletari ado\%20de\%20serviço\%20na\%20era\%20digital\&f=f alse

32. Antunes R. La centralidad del trabajo hoy. Papeles de población. 2000; 6(25):83-96. Disponible en:

http://www.scielo.org.mx/scielo.php?script=sci_art text\&pid=S1405-74252000000300005

33. Neffa J. Los riesgos psicosociales en el trabajo: contribución a su estudio. Buenos Aires: CONICET. $2015 . \quad$ Disponible en: http://biblioteca.clacso.edu.ar/Argentina/foumet/20160212070619/Neffa.pdf

34. Rentería E, Malvezzi S. Psicologías organizacionales y de los trabajos - POTs. Preguntas desde las realidades actuales del mundo del trabajo en América Latina. Psicología desde el Caribe, 
Número especial. 2018; 129-147. Disponible en: https://rcientificas.uninorte.edu.co/index.php/psic ologia/article/view/11756/214421443441

35. Cacciamali M. Globalização e processo de informalidade. Economia e sociedade. 2000; 9(1):153-174. Disponible en: https://periodicos.sbu.unicamp.br/ojs/index.php/e cos/article/view/8643124/10674

36. Costa M. Trabalho informal: um problema estrutural básico no entendimento das desigualdades na sociedade brasileira. Caderno Crh. 2010;23(58). Disponible en: http://www.scielo.br/pdf/ccrh/v23n58/v23n58a11. pdf

37. Organización internacional del trabajo. Medición de la economía informal. [internet]. [Consultado 2021 Abr 9]. Disponible en: https://www.ilo.org/wcmsp5/groups/public/--ed_emp/--emp_policy/documents/publication/wcms_229450 .pdf

38. Xavier D. Mobilização de competências na atividade informal do vendedor ambulante em praia de Natal (RN) (Mestrado dissertation, Universidade Federal do Rio Grande do Norte); 2014. Disponible em:

https://repositorio.ufrn.br/jspui/bitstream/123456 789/19769/1/DeboraGuerraPereiraXavier_DISSERT. pdf

39. Naciones Unidas. Informe de los objetivos de desarrollo sostenible 2020. [internet]. [Consultado 2021 Abr 9]. Disponible en: https://unstats.un.org/sdgs/report/2020/The-

Sustainable-Development-Goals-Report2020_Spanish.pdf

40. Rentería E, Malvezzi S, Giraldo A. Empleabilidad: inserción y movilidad en mercados de Trabajo de recién egresados de una universidad pública colombiana. En Carlos Mejía Sanabria y Deidi Maca Urbano. Paisajes laborales postfordistas en el suroccidente colombiano: organización y condiciones de trabajo en diferentes sectores de la economía. Cali: Programa Editorial Universidad del Valle; 2017. p 531 - 554. Disponible en: https://www.researchgate.net/publication/324653 914

41. Botero J, Rentería E. (2019). Empleabilidad y trabajo del profesorado universitario. Una revisión del campo. Athenea Digital. 2019 Nov;19(3):1-27. Doi: https://doi.org/10.5565/rev/athenea.2140

42. Enríquez A, Rentería E. Estrategias de aprendizaje para la empleabilidad en el mercado del trabajo de profesionales recién egresados. Universitas psychologica. 2006;6(1):89-103. Disponible en: https://revistas.javeriana.edu.co/index.php/revPsy cho/article/view/97

43. Organización Mundial del Turismo. ¿'Por qué el Turismo? 2019. [internet]. [Consultado $2021 \mathrm{Abr} 9$ ]. Disponible en: https://www.unwto.org/es/turismo

44. Presidencia de la República de Colombia. Bogotá, Bolívar y Valle del Cauca lideran ranking de competitividad turística. [internet]. [Consultado $2021 \quad$ Abr 12]. https://id.presidencia.gov.co/Paginas/prensa/2018 /181128-Bogota-Bolivar-y-Valle-del-Cauca-lideranranking-de-competitividad-turistica.aspx

45. Meisel-Roca A, Ricciulli-Marin D. La pobreza en Santa Marta: Los Estragos del Bien. Documento de Trabajo sobre Economía Regional y Urbana. 2018 abril; 266:1-73. Disponible en: http://repositorio.banrep.gov.co/handle/20.500.12 134/9184

46. Alves MA, Tavares MA. A dupla face da informalidade do trabalho: "Autonomia" ou precarização. En: Ricardo Antunes. Riqueza e miséria do trabalho no Brasil. São Paulo: Boitempo; 2006. p. 425-444.

47. Banister $P$, Burman $E$, Parker I, Taylor $M$, Tindall C. Métodos cualitativos en psicología. Centro Universitario de Ciencias de la Salud. Guadalajara: Universidad de Guadalajara; 2004. Disponible en: http://newpsi.bvspsi.org.br/ebooks2010/pt/Acervo_files/MetodosCu alitativos-completo.pdf 
48. Spink MJ, Lima H. Rigor e visibilidade: a explicitação dos passos da interpretação. En Mary Jane Spink. Práticas discursivas e produção de sentidos no cotidiano: aproximações teóricas e metodológicas. São Paulo: Cortez; 2013. p. 71-99.

49. Íniguez L. Métodos cualitativos de investigación en ciencias sociales: El debate sobre metodología cualitativa versus cuantitativa. 2008 . Disponible en: https://metodos.files.wordpress.com/2008/02/5_ metodologia-cualitativa.pdf

50. Burrell G, Morgan G. Sociological paradigms and organizational analysis.London: Aldershot, Gower; 1979.

51. Moran D. Introducción a la fenomenología. Barcolona, España: Anthropos Editorial; 2011. Disponible en: https://scholar.google.com/citations?view_op=vie w_citation\&hl=th\&user=RDyHfngAAAAJ\&citation_f or_view=RDyHfngAAAAJ:PR6Y55bgFSsC

52. Yin R. Applications of Case Study Research. London: Sage; 2012. Disponible en: https://books.google.es/books?hl=es\&lr=\&id=FgSV OY2FleYC\&oi=fnd\&pg=PP1\&dq=Case+Study+Resear ch+yin\&ots=4396RkwjPq\&sig=WaeHrg_AZ2EpeTnP bBh4EmVhPgw\#v=onepage \&q=Case\%20Study\%20 Research\%20yin\&f=false

53. Pinheiro O. Entrevista: uma Prática Discursiva. En Mary Jane Spink. Práticas discursivas e produção de sentidos no cotidiano: aproximações teóricas e metodológicas. São Paulo: Cortez; 2013. p. 156-187.

54. Bonilla $E$, Rodríguez P. La investigación en ciencias sociales: más allá del dilema de los métodos. Bogotá: Norma; 2000.

55. Bardin L. Análisis de contenido. Madrid: Akal; 2002.

56. Spink MJ, Medrado B. Produção de Sentido no Cotidiano. En Mary Jane Spink. Práticas discursivas e produção de sentidos no cotidiano: aproximações teóricas e metodológicas. São Paulo: Cortez; 2013. p. 22-41.
57. Suárez C, Del Moral G, González M. Consejos prácticos para escribir un artículo cualitativo publicable en Psicología. Psychosocial Intervention. 2013;22(1):71-79.

Doi: http://dx.doi.org/10.5093/in2013a9.

58. Naciones Unidas. Informe de los objetivos de desarrollo sostenible 2019. [internet]. [Consultado 2021 Abr 19]. Disponible en: https://unstats.un.org/sdgs/report/2019/The-

Sustainable-Development-Goals-Report2019_Spanish.pdf

59. Cárdona, LM. Economía Informal: Vendedores de transporte urbano. Ruído Blanco. 2003;1:49-61.

60. Andrade V, Bolaños C, Castrillón MJ. Empleabilidad en la economía informal, una investigación cualitativa con manicuristas de alta empleabilidad. Pensamiento psicológico. 2011 Feb;9(16):69-88. Disponible en: https://www.redalyc.org/pdf/801/80118612005.pd f

61. Busso M. Le travail informel: entre théorie et expérience. $2005 . \quad$ Disponible en: https://halshs.archives-ouvertes.fr/halshs00087363/document

62. Salanova M, Gracia F, Peiró J. Significado del trabajo y valores laborales. Tratado de psicología del trabajo. 1996; 2.35-63.

63. Xavier D, Falcão J, Torres C. Caracterização da atividade laboral de trabalhadores informais em praia de Natal (RN) - Brasil. Cadernos de Psicologia Social do Trabalho. 2015;18(1):29-45. Doi:10.11606/issn.1981-0490. v18n1p29-45

64. Sato L. Visitando a feira livre: notas sobre sua organização e seu trabalho. Mnemosine. 2009; 5(2):227-248. Disponible en: https://www.epublicacoes.uerj.br/index.php/mnemosine/article/ view/41441/28710

65. Sato L. Psicologia e trabalho: focalizando as profissões ignoradas. Psicologia Social e seus movimentos. 2011; 30:233-249. Disponible en: https://edisciplinas.usp.br/pluginfile.php/3396584/ 
mod_resource/content/1/SATO\%2C\%2OL.\%2OPsico logia\%20e\%20trabalho-

\%20focalizando\%20as\%20profissões\%20ignoradas.

pdf

66. Álvaro J. Desempleo y Bienestar Psicológico.

Madrid: Siglo XXI; 1992. 